\title{
Imiquimod $5 \%$ bei großem Rumpfhautbasaliom an der Rückenhaut - eine Therapieoption für pflegebedürftige Patienten
}

\author{
Imiquimod 5\% for an Extensive Basal Cell Carcinoma - A Therapeutic Option for Patients in Need of Care
}

Autoren

Institute

\section{Bayerl ${ }^{1}$, V. Jansen ${ }^{2}$, P. Paraskevopoulos ${ }^{3}$}

Klinik für Dermatologie und Allergologie Wiesbaden, HSK, Wilhelm Fresenius Klinik GmbH, Lehrkrankenhaus der Universität Mainz

Hautarztpraxis Dermatologie, Allergologie, Umweltmedizin, Mannheim

Klinik für Dermatologie, Venerologie und Allergologie, Klinikum Mannheim gGmbH

\section{Bibliografie}

DOI $10.1055 / \mathrm{s}-2007-966126$

Akt Dermatol 2007; 33:

79-81 (c) Georg Thieme

Verlag KG Stuttgart · New York ISSN 0340-2541

Korrespondenzadresse

Prof. Dr. med. Christiane BayerI

Klinik für Dermatologie und Allergologie Wiesbaden HSK, Wilhelm Fresenius Klinik $\mathrm{GmbH}$, Lehrkrankenhaus der Universität Mainz Aukammallee 39 65191 Wiesbaden christiane.bayerl@hskwiesbaden.de

\section{Zusammenfassung \\ $\nabla$}

Es stehen vielfältige therapeutische Möglichkeiten zur Behandlung des Basalzellkarzinoms (BCC) zur Verfügung. Bei älteren, bettlägerigen Patienten stellt sich die Frage, welcher für den individuellen Patienten der beste therapeutische Weg ist. Körperstellen, die auf der Matratze aufliegen wie der Rücken und die Rückseite der Beine und Arme zeigen eine schlechtere Wundhei-

\section{Einleitung \\ $\nabla$}

Das Basaliom ist ein destruierend wachsender Hauttumor, der in der Regel nicht metastasiert. Das Standardvorgehen ist die operative Sanierung mit histologischer Randschnittkontrolle. Weitere therapeutische Optionen für das Rumpfhautbasaliom umfassen Strahlentherapie (Röntgenoberflächenbestrahlung), Kryotherapie, Lasertherapie, Photodynamische Therapie und lokale medikamentöse Behandlungen mit 5-Fluorouracil oder Imiquimod 5\% [1]. Bei der topischen Applikation von Imiquimod beim Rumpfhautbasaliom wird ein Ansprechen bei $87 \%$ berichtet [2].

Imiquimod (1-(2-methylpropyl)-1H-imidazo (4,5-)quinolin-4 amin) ist ein topischer Immunmodulator, der die zelluläre Immunantwort stimuliert und dadurch indirekt antiviral wirkt und einen Anti-Tumor-Effekt zeigt [3]. Imiquimod induziert Interferon $\alpha$, Interleukine (IL-1, IL-5, IL-6, IL-8, IL-10, IL-12), Tumornekrosefaktor (TNF)- $\alpha$, den Interleukin-1 Rezeptorantagonisten (IL1RA), den Granulozyten-Kolonie-stimulierenden Faktor (G-CSF), den Granulozyten-Makrophagen/ Kolonie-stimulatierenden Faktor (GM-CSF), die Makrophagen inflammatorischen Proteine $1 \alpha$ und $1 \beta$ (MIP-1 $\alpha$ und MIP-1 $\beta$ ) und das Makrophagen chemotaktische Protein (MCP-1) $[4,5]$. lung und ein höheres Infektionsrisiko nach Operationen oder anderen therapeutischen Maßnahmen. Wir berichten über eine 84-jährige Patientin mit einem ausgedehnten Rumpfhautbasaliom am Rücken. Eine Behandlung mit Imiquimod 5\% über 4 Monate wurde gut angenommen, vertragen und erwies sich als sicher und histologisch kontrolliert auch als effektiv. Imiquimod 5\% ist gerade für bettlägerige Patienten eine Therapieoption für das Rumpfhautbasaliom.

\section{Kasuistik \\ $\boldsymbol{\nabla}$}

\section{Anamnese und Befund}

Bei Vorstellung im Juni 2003 zeigte eine 84-jährige Patientin an der Rückenhaut einen $9 \times 7 \mathrm{~cm}$ messenden livid roten Herd mit perlschnurartigem Randsaum. Die Patientin saß im Rollstuhl und wurde von ihrer Familie häuslich gepflegt. An Grunderkrankungen war eine Hypertonie bekannt. Es wurde Concor 5 plus ${ }^{\circledR}$ eingenommen. An größeren Operationen war 1969 eine Hysterektomie erfolgt, bei der sich kein maligner Prozess ergab. Zur Zeit war die Patientin den Großteil des Tages bettlägrig.

\section{Diagnostik}

Eine Stanze an der Rückenhaut aus dem fraglichen Prozess durch die zuweisende Dermatologin im Mai 2003 zeigte in der basalen Epidermis basaloide Tumorzellkomplexe mit Palisadenbildung der äußersten Zellreihe und umgebendes myxoides Stroma. Diagnose: oberflächliches Basalzellkarzinom.

\section{Therapie und Verlauf \\ $\nabla$}

Nach Analyse der Vor- und Nachteile der verschiedenen therapeutischen Optionen (Operation, Laser, Radiotherapie, PDT, Imiquimod 5\%) 


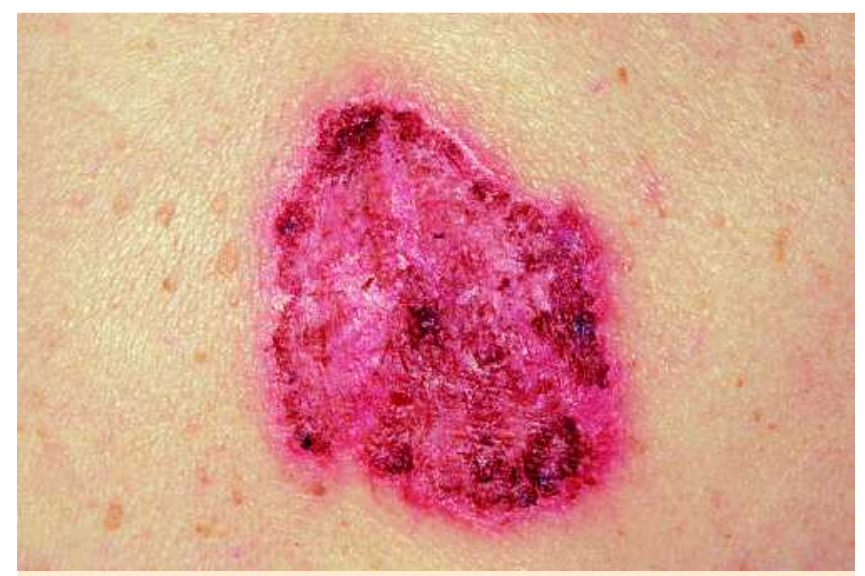

Abb. 1 Rumpfhautbasaliom an der Rückenhaut einer 84-jährigen Patientin nach der vierten Behandlungswoche mit Imiquimod $5 \%$; zentral wird die Stelle nach Biopsieentnahme zur Diagnosesicherung sichtbar.

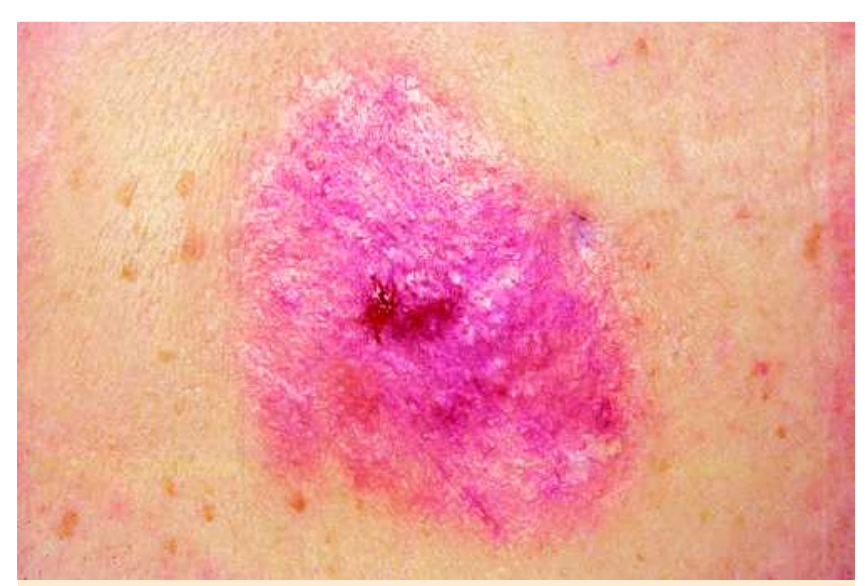

Abb. 2 Befund nach 8-wöchiger topischer Applikation von Imiquimod $5 \%$.

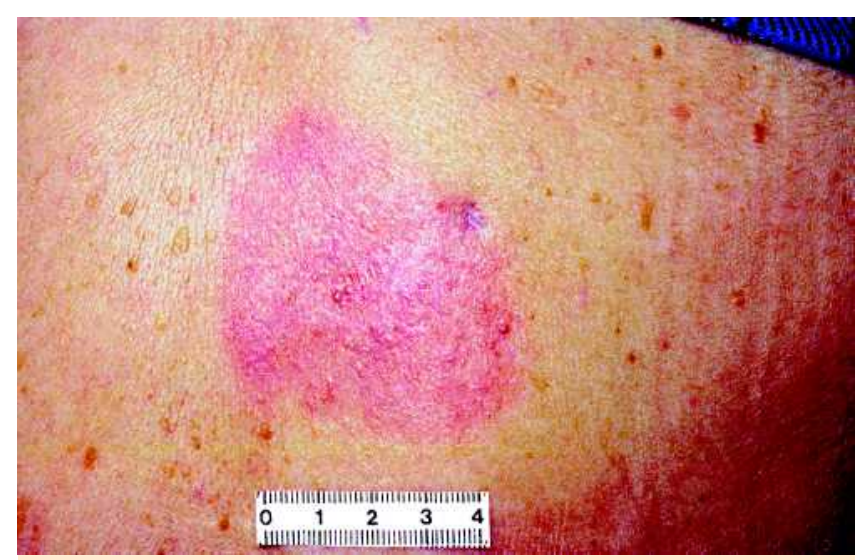

Abb. 3 Am Therapieende nach 16 Wochen ist histologisch kein Basaliom mehr nachweisbar. Es zeigt sich die deutliche entzündliche Begleitreaktion.

fiel die Entscheidung für eine Behandlung mit 5\% Imiquimod Creme, Start Juni 2003. Die Applikation wurde von der Tochter täglich abends durchgeführt in der Stunde, in der die Patientin zum Abendessen am Tisch saß. Anfangs wurde kein Pflaster an der Rückenhaut aufgebracht. Bei der Wiedervorstellung nach 10 Tagen zeigt sich eine entzündliche Reaktion und leichte Erosionen. Für die topische Behandlung am Morgen wurde Fucidine
Creme rezeptiert. Bei der Kontrolle nach 3 Wochen im Juli 2003 waren die erosiven Areale im Rumpfhautbasaliom etwas ausgeprägter. Es wurden keine Schmerzen angegeben; Fieber war nicht aufgetreten. In der vierten Behandlungswoche war nur noch der Randbereich leicht erosiv ( $\bullet$ Abb. 1). Die Behandlung wurde auf 3-mal pro Woche reduziert. Ein Pflaster wurde aufgebracht. Bei der Vorstellung im August 2003 zeigte sich bei weiterhin guter Verträglichkeit eine $7 \times 5,5 \mathrm{~cm}$ messende leicht gerötete Makel ( $\bullet$ Abb. 2). Im September 2003 maß der Herd noch $6 \times 5 \mathrm{~cm}$. Die Behandlung wurde nun 3-mal pro Woche fortgesetzt. Im Oktober 2003 ( $\odot$ Abb. 3) wurde eine 6 mm Stanzbiopsie zur Therapiekontrolle durchgeführt. Es zeigte sich eine schüttere gemischtzellige subepidermale Entzündungsreaktion mit geringgradig entzündlich bedingter Ödemisation des gut kapillarisierten subepidermalen Bindegewebes ohne Nachweis von Residuen des vorbehandelten Basalioms. Eine Kontrolle in 3 Monaten wurde empfohlen.

\section{Diskussion}

Bei einem Pflegefall mit Rumpfhautbasaliom am Rücken untersuchten wir die topische Wirksamkeit und Toleranz von Imiquimod, einem topischen „Immune response modifier“, der die Produktion von Interferon- $\alpha$ und anderer Zytokine beeinflusst.

Ein Nachteil der nicht-operativen Therapien beim Basaliom ist die fehlende histologische Kontrolle. So lag bei 3-mal wöchentlicher Behandlung mit Imiquimod unter Okklusion die Erfolgsrate bei superfiziellen BCC bei $87 \%$, bei nodulären bei $65 \%$ [2]. Bessere Ansprechraten sind bei täglicher Imiquimod Anwendung über 6 Wochen beschrieben [6]. Die Zulassung für Imiquimod $5 \%$ bezieht sich auf eine einmal tägliche Anwendung an 5 Tagen in der Woche [7]. Augrund der Größenausdehnung des Tumors und des Risikos der Wundheilungsstörung bei Bettlägerigkeit der Patientin fiel die Entscheidung gegen ein operatives oder Kryo-therapeutisches Vorgehen und gegen eine Behandlung mit dem $\mathrm{CO}_{2}$-Laser. Mehrfach fraktionierte Röntgenweichteilbestrahlungen mit niedrigen Einzeldosen und mehr als 10 Sitzungen wurden als zu zeitaufwendig gewertet. Einer photodynamischen Therapie stimmte die Patientin aufgrund der Schmerzhaftigkeit und der ungeklärten Frage der Kostenübernahme nicht zu. Die topische Immuntherapie mit dem Chemotherapeutikum 5-Fluorouracil führt zu starker entzündlicher Reaktionen im Vergleich zum Konkurrenzpräparat Imiquimod [8] und wurde daher zurückgestellt.

Bei unserer Patientin waren eine entzündliche Begleitreaktion und Erosionen im Behandlungsfeld aufgetreten, die aber keine Beeinträchtigung für sie darstellten. Das Ausbleiben systemischer Nebenwirkungen in der Kasuistik korrespondiert mit den Berichten über geringe systemische Aufnahmen des Imiquimod. Bei Urinuntersuchungen wurde gezeigt, dass weniger als $1 \%$ nach einmaliger Applikation nachgewiesen wurde [5]. Die am häufigsten beschriebenen Nebenwirkungen unter Imiquimod sind Erythem, Juckreiz, Brennen oder Schmerzen. Systemische Nebenwirkungen traten nicht auf, insbesondere keine grippeähnlichen Beschwerden, kein Fieber, keine Muskelschmerzen oder Kopfschmerzen.

Imiquimod 5\% (Aldara ${ }^{\mathrm{TM}}$ ) ist zugelassen für die lokale Behandlung der externen genitalen und perianalen Condylomata acuminata [9]. Andere virale Infektionen, Verrucae planae [10], Verrucae vulgares [11] und Mollusca contagiosa [12] zeigen mitunter Ansprechen auf Imiquimod. Bezüglich der Behandlung von 
Tumoren ist die Substanz nicht nur beim Basaliom effektiv [13], sondern auch bei aktinischen Keratosen [14] und intraepithelialen Neoplasien [15]. Bei Lentigo maligna [16], Morbus Bowen [17] und interessanterweise auch bei parasitären Erkrankungen wie der kutanten Leishmanios [18] gibt es Berichte über Erfolge. Bei unserer Patientin konnte ein chirurgisches Vorgehen vermieden werden. Die Therapie mit Imiquimod hat den Vorteil, weniger traumatisierend für alte Menschen zu sein als die üblichen Behandlungsoptionen bei BCC, die als schmerzhaft und angsteinflößend empfunden werden können. Andererseits erfordern die lange Behandlungszeit und die begleitende lokale Entzündung eine gute Patientenführung und die Motivation der Familien oder Pflegekräfte.

\section{Fazit für die Praxis \\ $\nabla$}

Das Rumpfhautbasaliom ist aufgrund seiner superfiziellen Natur besonders geeignet für eine lokale Immuntherapie. Imiquimod $5 \%$ Creme stellt für pflegebedürftige Patienten eine Therapieoption dar. Die betreuende Person übernimmt die tägliche Applikation. Das Vorgehen erspart den meist älteren Patienten Angst vor einer Operation, Wundheilungsstörungen, Transport und häufige Konsultationen. Die entzündliche Begleitreaktion nach Imiquimod wird trotz Bettlägerigkeit gut toleriert.

\section{Abstract}

\section{Imiquimod 5\% for an Extensive Basal Cell Carcinoma - A Therapeutic Option for Patients in Need of Care}

There is a wide variety of topical or surgical treatment options for basal cell carcinoma (BCC). The decision which might be the best advice for the individual treatment of an old patient confined to bed is difficult. Body sides that rest on the mattress such as the back or the backsides of leg and arms may show slower healing and have a higher risk of infections after surgery. In the presented case report about an extensive superficial BCC on the back of an 84-year-old women, good acceptance, tolerability, safety and histologically controlled complete remission were achieved after daily application of Imiquimod $5 \%$ for 4 months. Imiquimod $5 \%$ should therefore be suggested as a treatment option for superficial BCC in patients confined to bed.

\section{Literatur}

1 AWMF-interdisziplinäre Leitlinie der Deutschen Krebsgesellschaft und der Deutschen Dermatologischen Gesellschaft. Basalzellkarzinom. http://www.uni-duesseldorf.de/WWW/AWMF/II/032 - 021.htm

2 Sterry W, Ruzicka T, Herrera E, Takwale A, Bichel J, Andreas K, Ding L, Thissen MR. Imiquimod $5 \%$ cream for the treatment of superficial and nodular basal cell carcinoma: randomized studies comparing low-frequency dosing with and without occlusion. Br J Dermatol 2002; 147: $1227-1236$

3 Dahl MV. Imiquimod: An immune response modifier. J Am Acad Dermatol 2000; 43: 1-5

4 Imbertson LM, Beaurline JM, Couture AM, Gibson SJ, Smith RMA, Miller $R L$. Cytokine induction in hairless mouse and rat after topical application of the immune response modifiers, imiquimod. J Invest Dermatol 1998; 110: $734-739$

5 Sauder DN. Immunomodulatory and pharmacologic properties of imiquimod. J Am Acad Dermatol 2000; 43: 6-11

6 Shumack S, Robinson J, Kossard S, Golitz L, Greenway H, Schroeter A, Andres $K$, Amies M, Owens $M$. Efficacy of topical $5 \%$ imiquimod cream for the treatment of nodular basal cell carcinoma: comparison of dosing regimens. Arch Dermatol 2002; 138: 1165- 1171

7 Geisse J, Caro I, Lindholm J, Golitz L, Stampone P, Owens M. Imiquimod $5 \%$ cream for the treatment of superficial basal cell carcinoma: results from two phase III, randomized, vehicle-controlled studies. J Am Acad Dermatol 2004; 50: $722-733$

8 Tsuji T, Otake N, Nishimura M. Cryosurgery and topical fluorouracil: a treatment method for widespread basal cell epithelioma in basal cell nevus syndrome. J Dermatol 1993; 20: 507-513

9 Centers for Disease Control and Prevention (CDC). Sexually transmitted diseases treatment guidelines. MMWR Recomm Rep 2002; 51: 1 - 78

10 Oster-Schmidt C. Imiquimod: a new possibility for treatment-resistant verrucae planae. Arch Dermatol 2001; 137: 666 - 667

11 Hengge UR, Esser S, Schultewolter T, Stockfleth E, Meyer T, Arndt R, Goos $M$. Self-administered topical imiquimod for the treatment of common warts and mollusca contagiosa. Br J Dermatol 2000; 143: 1026-1031

12 Bayerl C, Feller G, Goerdt S. Experience in treating molluscum contagiosum in children with imiquimod 5\% cream. Br J Dermatol 2003; 149 Suppl 66: $25-29$

13 Marks R, Gebauer K, Shumack S, Amies M, Bryden J, Fox TL, Owens ML. Imiquimod $5 \%$ cream in the treatment of superficial basal cell carcinoma: results of a multicenter 6-week dose-response trial. J Am Acad Dermatol 2001; 44: 807-813

14 Stockfleth E, Meyer T, Benninghoff B, Christophers E. Successful treatment of actinic keratosis with imiquimod cream $5 \%$ : a report of six cases. Br J Dermatol 2001; 144: 1050 - 1053

15 Diaz Arrastia C, Arany I, Robazeti SC, Dinh TV, Gatalica Z, Tyring SK, Hanningan E. Clinical and molecular responses in high-grade intraepithelial neoplasia treated with topical imiquimod $5 \%$. Clin Cancer Res 2001; 7: $3031-3033$

16 Ahmed I, Berth-Jones J. Imiquimod, a novel treatment for lentigo maligna. Br J Dermatol 2000; 143: 843-845

17 Mackenzie-Wood A, Kossard S, de Launey J, Wilkinson B, Owens ML. Imiquimod $5 \%$ in the treatment of Bowen's disease. J Am Acad Dermatol 2001; 44: $462-470$

18 Arevalo I, Ward B, Miller R, Meng TC, Najar E, Alvarez E, Matlashewski G, Llanos-Cuentas A. Successful treatment of drug-resistant cutaneous leishmaniasis in humans by use of imiquimod, an immunomodulator. Clin Infect Dis 2001; 33: 1847-1851 